\title{
Extracción endoscópica de cuerpos extraños digestivos
}

\author{
GLORIA RÍOS M. ${ }^{1}$, FRANCISCO ALLIENDE G. ${ }^{1}$, ISABEL MIQUEL E. ${ }^{1}$, \\ MARÍA EUGENIA ARANCIBIA S. ${ }^{1}$, LORENA RODRÍGUEZ G. ${ }^{1}$, ERIC SAELZER W. ${ }^{1}$ \\ 1. Unidad de Gastroenterología, Departamento de Pediatría. Clínica Alemana de Santiago. Facultad de Medicina, \\ Clínica Alemana Universidad del Desarrollo. Santiago. Chile.
}

\begin{abstract}
Endoscopic extraction of digestive foreign bodies

Introduction: The ingestion of a foreign body (FB) is very common in childhood, 10 to $20 \%$ of the cases require endoscopic removal. The aim of this study is to analyze the demographic, clinical and endoscopic characteristic of patients with FB ingestion requiring endoscopic management. Patients and Methods: The design corresponded to a Prospective Cohort Study. It included patients referred to the Clinica Alemana ER in Santiago in order to remove foreign bodies from their digestive systems between November 2007 and November 2011. Demographic, clinical and endoscopic characteristics were recorded, classifying the symptoms as respiratory, digestive and other manifestations. Risk factors and complications were evaluated. Results: 170 patients, $52.4 \%$ male with average age of four years and three months old. In $78.5 \%$ of the cases, the FB ingestion occurred at home, $82.1 \%$ sought medical attention within two hours from the ingestion. Digestive, respiratory and local pain were among the most common symptoms. The location of the FB took place using simple $x$-ray in $87.6 \%$ of the cases. $91.8 \%$ of the FBs were extracted. Location: retropharyngeal $1.3 \%, 69.3 \%$ esophagus, stomach $23 \%$, duodenum $1.9 \%$ and $3.8 \%$ distal duodenum. Round FB were the most frequent $(56.4 \%)$ like coins, sharp objects $(24.1 \%)$, batteries $(14.7 \%)$, magnets $(2.9 \%)$ and other large items $(1.8 \%)$. Mucosal lesion was found in $53.5 \%$ of the cases and $9.8 \%$ required hospitalization Conclusions: The ingestion of FB occurred at home, and it is most frequent in preschool children. The most common FB was a coin. The location of the FB was through x-ray and no failure or complications were described during the endoscopic removal.
\end{abstract}

(Key words: Foreign body, endoscopic extraction, pediatric age, accidents).

Rev Chil Pediatr 2013; 84 (5): 505-512

\section{RESUMEN}

Introducción: La ingestión de cuerpo extraño (CE) es frecuente en la infancia, requiriendo en el 10-20\% de los casos extracción endoscópica. El objetivo de este trabajo fue analizar las características demográficas, clínicas y endoscópicas de los pacientes con ingesta de CE que requieren manejo endoscópico. Pacientes y

Recibido el 4 de mayo de 2012, devuelto para corregir el 5 de septiembre de 2012, segunda versión 9 de enero de 2013, tercera versión 5 de junio de 2013, aceptado para publicación el 17 de julio de 2013.

Este trabajo cumple con los requisitos sobre consentimiento /asentimiento informado, comité de ética, financiamiento, estudios animales y sobre la ausencia de conflictos de intereses según corresponda. 
Método: Estudio de cohorte prospectivo. Se incluyen los pacientes derivados al servicio de urgencia de Clínica Alemana de Santiago para extracción de cuerpo extraño entre noviembre de 2007-noviembre de 2011. Se registran variables demográficas, clínicas y endoscópicas, clasificando la sintomatología en respiratoria, digestiva y otros. Se evaluaron factores de riesgo y complicaciones. Resultados: 170 pacientes, $52,4 \%$ varones, edad promedio de 4 años y 3 meses. En 78,5\% la ingestión de CE ocurrió en el hogar, el 82,1\% consultó en las primeras $2 \mathrm{~h}$. Los síntomas más comunes post ingestión fueron digestivos, respiratorios y dolor local. La localización del CE fue con radiografía simple en 87,6\%. En el 91,8\% se extrajo el CE. Localización: 1,3\% retrofaríngeo, $69,3 \%$ esófago, $23 \%$ estómago, 1,9\% duodeno, 3,8\% distal a duodeno. Los CE más frecuentes fueron los redondos $(56,4 \%)$ y dentro de ellos las monedas, punzantes $(24,1 \%)$, pilas $(14,7 \%)$, imanes $(2,9 \%)$ y otros de gran tamaño (1,8\%). Se encontró lesión de mucosa en el 53,5\%, requiriendo hospitalización el 9,8\% de los niños. Conclusiones: La ingestión de CE ocurre en el hogar y es más frecuente en pre escolares. El CE más frecuentes fue la moneda. La localización del CE fue con radiografía simple y no hubo fracaso ni complicaciones en la extracción endoscópica.

(Palabras clave: Cuerpo extraño, extracción endoscópica, pediatría, accidentes).

Rev Chil Pediatr 2013; 84 (5): 505-512

\section{Introducción}

La ingestión accidental de cuerpo extraño (CE) es frecuente en los niños, ya que como parte de su desarrollo se llevan a la boca toda clase de objetos ${ }^{1}$. En un estudio de seguimiento de niños sanos realizado en Estados Unidos, se encontró que alrededor de un 4\% de los niños se traga una moneda en los primeros años de vida ${ }^{2}$. En el año 2009 se reportaron al "American Poison Control Centers" 125.179 casos de ingestión de CE en menores de 19 años $^{3}$. Si bien la ingestión de CE constituye un motivo de consulta en servicios de urgencia, solo un $10-20 \%$ de los casos requiere una resolución endoscópica y en forma excepcional tratamiento quirúrgico ${ }^{4,5}$. Las complicaciones graves como perforaciones, fístulas a órganos vecinos ó grandes vasos, abscesos, u otros, son poco frecuentes pero implican riesgo vital ${ }^{6-10}$.

El tipo de CE ingerido por los niños varía de un país a otro, dependiendo de factores culturales, ambientales y alimentarios ${ }^{11-14}$. En nuestro país no se cuenta con grandes series pediátricas publicadas.

Existen diversas pautas de manejo de $\mathrm{CE}$ digestivos donde se consideran básicamente 3 aspectos: presencia de síntomas, características del cuerpo extraño (tamaño, forma y composición) y localización del $\mathrm{CE}^{15-17}$.

El objetivo de este estudio fue evaluar las características demográficas, clínicas y endoscópicas de pacientes pediátricos que ingresados al Servicio de Urgencia de Clínica Alema- na (SUCA) para extracción de cuerpo extraño digestivo.

\section{Pacientes y Método}

Estudio de Cohorte prospectivo. Se ingresaron los pacientes referidos a SUCA para extracción de CE, entre noviembre de 2007 y noviembre de 2011, provenientes de centros hospitalarios públicos y privados. Todos los pacientes fueron evaluados por un residente pediátrico de urgencia. La conducta seguida en cada caso se basó en la Pauta de Manejo de CE Digestivo, documento interno de Clínica Alemana de Santiago (CAS) (tabla 1). Se registraron los datos demográficos, clínicos y endoscópicos de acuerdo a un protocolo preexistente, los que luego se llevaron a una base de datos. Los síntomas se agruparon en respiratorios (tos, crisis de cianosis, estridor, dificultad respiratoria), digestivos (disfagia, sialorrea, vómitos, sensación de presencia de CE, rechazo de alimentación) dolor local y otros (hematemesis, petequias faciales post esfuerzo).

Se registró la presencia de factor de riesgo de complicación, como la preexistencia de patología esofágica. La endoscopía digestiva alta (EDA) fue realizada por miembros entrenados de la Unidad de Gastroenterología Infantil de CAS, bajo sedación administrada por anestesiólogo pediátrico.

Se consideró complicación de la ingestión 
Tabla 1. Pauta de extracción de cuerpo extraño digestivo alto (CAS)

\begin{tabular}{|c|c|c|}
\hline Tipo de CE & En esófago & En estómago y duodeno \\
\hline Moneda & $\begin{array}{l}\text { Siempre remover } \\
\text { Sintomático: urgente } \\
\text { Asintomático: antes de } 24 \mathrm{~h} \\
>\text { de } 2 \mathrm{~cm} \text { extraer precozmente. }\end{array}$ & $\begin{array}{l}\text { Observar hasta por 3-4 semanas } \\
\text { Mayores de } 2 \mathrm{~cm} \text { considerar extracción precoz }\end{array}$ \\
\hline Pilas tipo botón & Extracción urgente & Observar hasta $48 \mathrm{~h}$ \\
\hline Corto punzante & Extracción urgente & Extracción urgente \\
\hline Alimento & $\begin{array}{l}\text { Sintomático: Extracción urgente } \\
\text { Asintomático: Antes de } 12 \mathrm{~h}\end{array}$ & \\
\hline CE grandes* & Extracción programada & Extracción programada \\
\hline Imán & $\begin{array}{l}\text { Si son } 2 \text { o más extraer urgente } \\
\text { Si es } 1 \text { extracción programada evitar contacto con } \\
\text { artículos metálicos externos }\end{array}$ & $\begin{array}{l}\text { Si son } 2 \text { o más extraer urgente } \\
\text { Si es } 1 \text { extracción programada Evitar contacto con } \\
\text { artículos metálicos externos }\end{array}$ \\
\hline Droga & No Endoscopia & No endoscopia \\
\hline
\end{tabular}

${ }^{*} \mathrm{CE}$ grandes en lactantes: $>3 \times 2 \mathrm{~cm}$ y en preescolar y escolar: $>5 \times 2 \mathrm{~cm}$.

Tabla 2. Características demográficas de los pacientes

\begin{tabular}{|ll|}
\hline Variable & Descripción \\
\hline Edad & Promedio: 4 años 3 meses (1 mes a 14 años 9 m) \\
& Lactantes: 50 (varón: 21$)$, Preescolares 77 (varón: 44), Escolares: 43 (varón: 24) \\
& Masculino: $89(52,4 \%)$ \\
& Femenino: $81(47,6 \%)$ \\
Procedencia: & Hospitales públicos: $79(46,5 \%)$ \\
& CAS: $62(36,5 \%)$ \\
& Otros centros privados: $29(17,1 \%)$ \\
Lugar de Ingestión del CE & Su casa: $78,5 \%$, Otras casas: $11,5 \%$ \\
& Colegio: $4,9 \%$, Vía pública: $4,1 \%$, Restaurante: $0,6 \%$ \\
\hline
\end{tabular}

CAS: Clínica Alemana de Santiago.

del CE o del procedimiento cuando se encontró lesión que requirió manejo especial como ayuno, observación u otro examen o cuando el paciente debió hospitalizarse después de la recuperación de la sedación.

Se realizó el análisis de datos con estadística descriptiva con medidas de frecuencia, tendencia central y dispersión.

\section{Resultados}

Ingresaron 170 pacientes que requirieron evaluación endoscópica por ingestión de CE. Las características demográficas se muestran en la tabla 2.

E1 77,6\% de los pacientes presentó algún síntoma post ingestión del CE, los que generalmente estaban asociados, destacando síntomas digestivos $(44,2 \%)$, dolor local $(34,5 \%)$, síntomas respiratorios $(30,3 \%)$ y otros $(1,8 \%)$.

El tiempo entre la ingestión del CE y la consulta al Servicio de Urgencia se consignó en 155 pacientes. En la figura 1 se muestra el porcentaje de pacientes que consultan en los diversos lapsos de tiempo, destacando que el $82,1 \%$ consulta en las 2 primeras $h$.

En 65 casos $(39,6 \%)$ se intentaron maniobras de extracción del CE, las que en su mayoría fueron aplicadas en el hogar por el cuidador del niño. Compresión abdominal y/o torácica en $33,8 \%$, intento de extracción manual en $26,1 \%$, maniobras asociadas en $18,5 \%$, cam- 


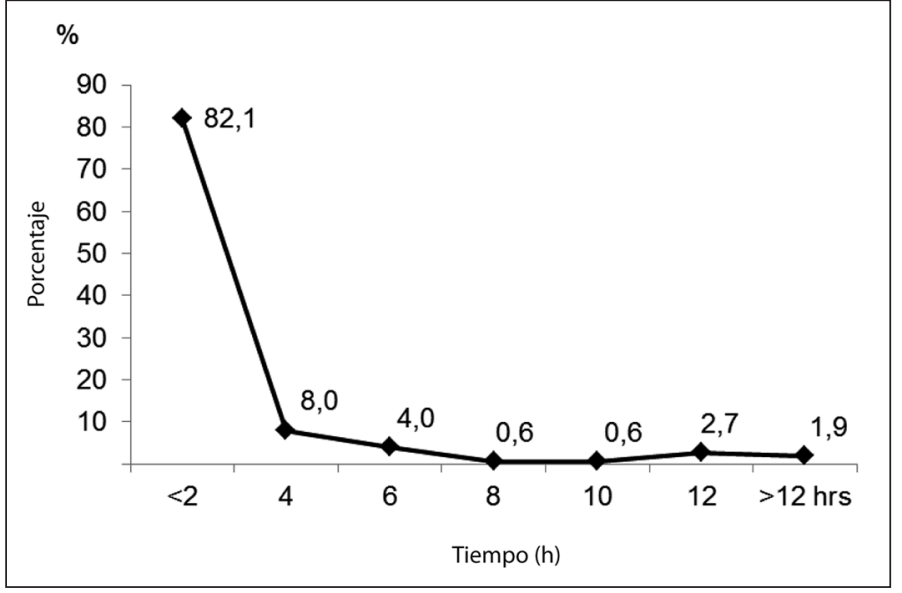

Figura 1. Tiempo transcurrido entre la ingestión del CE y consulta en Servicio de Urgencia. bio de posición en 13,8\%, administración de líquidos en 4,6\% y uso de pinza Magill por un médico en servicio de urgencia 3,0\%, siendo todas ellas fallidas.

El tipo de cuerpo extraño ingerido se describe en la tabla 3 siendo los más frecuentes los redondos y/o romos. E1 87\% eran radiopacos y en el $94,7 \%$ únicos. De los 96 objetos romos, $67(69,8 \%)$ fueron monedas, las que se detallan en la tabla 4 , siendo la de $\$ 10$, cuyo diámetro es de $21 \mathrm{~mm}$, la más frecuente $(55,2 \%)$. Los otros objetos redondos fueron botones, ruedas, argollas de llaveros, anillo, bolas de diversos materiales, tuerca, piedra, alimentos, carozo de guinda.

Los objetos corto punzantes de diversos orígenes, los agrupamos en: trozos de vidrio, plástico y discos compactos; tornillos, alfileres y agujas de costura; medallas y aros de forma irregular, artículos de ortodoncia; pinches; alimentos (espina pescado y huesos de pollo), alfileres de gancho; clips y ganchos y misceláneas.

En relación a las baterías, todas tipo botón y entre 7 y $20 \mathrm{~mm}$, estaban en desuso, fuera del dispositivo a que pertenecían, sin poder conocer su carga. Pertenecían a equipos o juegos electrónicos. Se extrajeron 22 de 25 pilas, 10 de esófago y 12 de estómago. De las 3 que no se extrajeron, 2 estaban distales al alcance del endoscopio y 1 no se encontró, pero si se observó una lesión mucosal secunadario a su paso.

Los imanes fueron de diversa forma y ta- maño (una flor, un botón y 3 imanes redondos pequeños); sólo en 1 caso se encontró 2 imanes juntos.

Del total de los CE, 20 (11,8\%) correspondían a artículos utilizados por niños, como juguetes redondos ${ }^{10}$, pinches ${ }^{3}$, alfileres de gancho $^{4}$ y medallas ${ }^{3}$. Los objetos ingeridos en el colegio fueron: alfileres en 2 casos, en uso en actividades escolares y los otros objetos fueron

Tabla 3. Características de cuerpos extraños ingeridos

\begin{tabular}{|lrc|}
\hline Característica & n & \% \\
\hline Romos-Redondos & 96 & 56,5 \\
\hline Corto punzante & 41 & 24,1 \\
\hline Pilas tipo botón & 25 & 14,7 \\
Imán & 5 & 2,9 \\
CE de gran tamaño & 3 & 1,8 \\
\hline Total & 170 & 100,0 \\
\hline
\end{tabular}

Tabla 4. Tipo y diámetro de monedas extraídas

\begin{tabular}{|c|c|c|c|}
\hline \multicolumn{2}{|c|}{ Tipo de moneda* y diámetro } & \multirow{2}{*}{$\begin{array}{c}\mathbf{n} \\
4\end{array}$} & \multirow{2}{*}{\begin{tabular}{|r}
$\%$ \\
6,0
\end{tabular}} \\
\hline$\$ 1$ y $5:$ & $15,5 \mathrm{~mm}$ & & \\
\hline$\$ 10:$ & $21,0 \mathrm{~mm}$ & 37 & 55,2 \\
\hline$\$ 50-100-500:$ & $23-27 \mathrm{~mm}$ & 20 & 29,9 \\
\hline \multicolumn{2}{|l|}{ No identificadas } & 6 & 8,9 \\
\hline \multicolumn{2}{|l|}{ Total } & 67 & 100,0 \\
\hline
\end{tabular}

*Monedas chilenas. 
monedas, juguetes y alimento. En 10 pacientes $(5,9 \%)$ el CE fue alimento impactado en esófago, de los cuales 4 fueron punzantes.

La tabla 5 detalla el método diagnóstico utilizado para determinar la localización del CE previo a la EDA. En el 86,5\% de los casos se logró identificar el CE con Rx simple; en el $38,8 \%$ de los casos fue de cuello, tórax y abdomen, en $46,3 \%$ se tomó Rx de 2 segmentos (cuello y tórax, o tórax y abdomen) y en 14,8\% de un solo segmento. Se usó radioscopia intra procedimiento en 9 casos y se pudo determinar que en 4 pacientes el CE estaba en estómago, entre restos de alimentos y en 5 distal al alcance del endoscopio.

En 4 pacientes se encontró factores de riesgo de impactación de CE: 3 con estenosis esofágica y 1 paciente con esofagitis eosinofílica (EoE).

La tabla 6 describe la localización del CE al momento de la EDA. En algunos casos se contó con apoyo fluoroscópico durante el procedimiento. El 63,5\% de los CE se encontraba en esófago ( $81,5 \%$ en esófago superior, $8,3 \%$ en medio y $10,2 \%$ en inferior).

El tiempo entre ingestión del CE y la extracción endoscópica fue muy variable y dependió del tipo de CE. El 95,3\% de los CE redondos se extrajo antes de las $31 \mathrm{~h}$ de ingeridos, existiendo 4 casos de consulta muy tardía, que se extrajeron entre las 96 y 174 h (4-7,25 días). El tiempo promedio fue de $14 \mathrm{~h}$, mediana 6 h (1,45 h a 174 h). En los demás CE no se observó tanta variación en los tiempos, correspondiendo en promedio a $6 \mathrm{~h} 40 \mathrm{~min}(2 \mathrm{~h}$ a 20 h $30 \mathrm{~min}$ ) para los punzantes, $5 \mathrm{~h} 22 \mathrm{~min}(2 \mathrm{a}$ $10 \mathrm{~h}$ ) para las baterías tipo botón y $3 \mathrm{~h} 42 \mathrm{~min}$ (1 h 30 min a 6 h) para los imanes.

Para la extracción del CE se usó diversos tipos de dispositivos, siendo los más frecuentes: pinza "diente de ratón" en 107 casos $(71,3 \%)$, pinza "caimán" en 19 (12,6\%), canastillo dormia en $8(5,3 \%)$, y malla en $4(2,6 \%)$. En 86 casos $(50,6 \%)$ se encontró lesiones asociadas al CE, 1 retro faríngea, 66 esofágicas, 18 gástricas y 1 duodenal. La presencia de lesión se relacionó principalmente con el tipo de $\mathrm{CE}$, observando lesión en todas las pilas impactadas en esófago y en el $82 \%$ de los objetos punzantes. La mayoría de las lesiones fueron
Tabla 5. Método diagnóstico utilizado para la localización del CE

\begin{tabular}{|lrc|}
\hline Método diagnóstico & n & \% \\
\hline Rx. Simple & 147 & 86,5 \\
Rx. Contrastada & 3 & 1,7 \\
\hline Clínica (+) y Endoscopia (+) & 18 & 10,6 \\
\hline Hallazgo de endoscopia & 2 & 1,2 \\
\hline Total & 170 & 100,0 \\
\hline
\end{tabular}

Tabla 6. Localización de cuerpo extraño digestivo

\begin{tabular}{|lrc|}
\hline Localización de CE & $\mathbf{n}$ & \% \\
\hline Retro faríngeo & 2 & 1.3 \\
Esófago & 108 & 63,5 \\
\hline Estómago & 37 & 21,8 \\
Duodeno & 3 & 1,7 \\
Distal duodeno & 6 & 3,5 \\
No encontrados & 14 & 8,2 \\
Total & 170 & 100,0 \\
\hline
\end{tabular}

superficiales como áreas congestivas o erosiones lineales por decúbito.

En 9 pacientes se indicó hospitalización y ayunas por la severidad del compromiso de la mucosa. De ellos, uno correspondía a un lactante de 1 mes, con una moneda alojada en tercio superior de esófago durante 1 semana y 8 pacientes por lesión esofágica secundaria a pila tipo botón. Los restantes 161 pacientes $(94,7 \%)$ fueron dados de alta, posterior a la recuperación de la sedación. No hubo complicaciones asociadas a la extracción del CE ni a la sedación en esta serie.

\section{Discusión}

En esta serie la ingestión de CE es un accidente que ocurre en el hogar, que afecta principalmente a menores de 6 años, sin diferencia en sexo en este grupo etario, y sintomática en el $77,6 \%$ de los casos. La edad de mayor frecuencia coincide con lo descrito en series de otros países ${ }^{18,19}$. Es destacable la presencia en nuestra serie de 2 lactantes de 1 y 3 meses que aún no tenían la capacidad de llevarse objetos a la boca. En ellos la ingestión posiblemente 
ocurrió mientras se les cambiaba ropa o bien por la intervención de otros niños. Los síntomas referidos son similares a los descritos en otras series ${ }^{11,19}$; cuando estos están presentes al momento de la endoscopía, habitualmente confirma la presencia del $\mathrm{CE}^{5}$, pero el estar asintomático no es sinónimo de su migración del CE hacia distal. En la literatura se ha reportado que entre el $51-84 \%$ de los casos, el accidente ocurre en presencia de un adulto ${ }^{20,21}$. Coincidimos con Paul y cols ${ }^{21}$, en que la presencia de un adulto no impide que el niño se lleve objetos a la boca.

El $82,1 \%$ de los casos consulta en forma precoz, lo que contrasta con la serie de Gregori y cols donde sólo el $25 \%$ de los padres consideró consultar rápidamente ${ }^{22}$. La consulta precoz de nuestra población (pública y privada) se puede explicar porque hay fácil acceso a servicios de urgencia, los niños estaban acompañados cuando tuvieron la impactación del CE, las maniobras de extracción en el hogar fueron fallidas, y no sabemos si en este sub grupo de pacientes que requirieron extracción del $\mathrm{CE}$, los síntomas fueron más alarmantes. Se plantea que la consulta tardía potencialmente conduciría a complicaciones ${ }^{22}$.

Por tratarse de un accidente, lo habitual es que los niños ingieran solo $1 \mathrm{CE}$, tal como lo vimos en nuestra serie. Cuando se encuentra más de $1 \mathrm{CE}$ se debe investigar la posibilidad de una forma de maltrato infanti123,24. Encontramos $2 \mathrm{CE}$ en el 5,3\% sin sospecha de mal trato infantil.

Tokar y cols, reporta que niños con alteraciones anatómicas esofágicas presentan 8,5 veces más complicaciones, por lo que es importante tener presente este antecedente ${ }^{25}$. En esta serie encontramos sólo 3 pacientes con atresia esofágica operada y cursaron sin complicaciones. El paciente portador de EoE presentó 2 episodios de impactación de alimentos. Se debe sospechar este cuadro frente a escolares, generalmente varones, con impactación de alimentos en esófago, sin una alteración anatómica. En estos casos se recomienda tomar biopsias escalonadas del esófago ${ }^{26,27}$. Nosotros no aplicamos esta norma en forma rutinaria por lo que podemos estar sub diagnosticando la EoE.

El método diagnóstico en la mayoría de los casos evaluados $(86,5 \%)$ fue la radiografía simple. Se recomienda que este examen incluya cuello, tórax y abdomen en 2 posiciones AP y Lateral ${ }^{5,15}$. Cuando el CE es radiolúcido se han planteado otros posibles exámenes como Rx contrastada, tomografía computada y evaluación endoscópica ${ }^{5}$. El uso de medio de contraste es controversial ya que dificultaría la visión del cuerpo extraño durante la EDA y existiría riesgo de aspiración si el esófago se encuentra obstruido ${ }^{15}$. En nuestra serie, en 3 casos se realizó estudio contrastado, utilizando una pequeña cantidad de bario diluido, sin observar complicaciones ni dificultad en la extracción del CE. Para evitar el riesgo de aspiración se recomienda usar esta técnica sólo en pacientes capaces de deglutir y controlar el volumen de bario a administrar.

Coincidente con la literatura, la mayoría de los CE estaban impactados en esófago, y dentro de él, en esófago superior ${ }^{11,28}$. Esto se debe a que el esófago es la zona más angosta del tracto gastrointestinal y tiene 3 estrecheces fisiológicas que dificultan el avance del $\mathrm{CE}^{29}$.

El CE más frecuente fue la moneda, representando el 39,4\% del total de los CE, situación similar a lo observado en series europeas y americanas ${ }^{17,30}$. Se han descrito varias técnicas para extraerlas ${ }^{31,32}$. Lo recomendable es realizar la técnica que cada centro domine, de acuerdo a sus medios y disponibilidad de equipos. Nosotros realizamos la extracción endoscópica utilizando las pinzas dientes de ratón o pinzas caimán con buenos resultados, sin observar complicaciones ni fracaso del procedimiento. Aunque el $94,7 \%$ de las monedas se extrajeron en el plazo estipulado por las diversas pautas ${ }^{15-17}$, en el $53,7 \%$ ya se evidenciaba daño en la mucosa.

Los objetos punzantes son los segundos en frecuencia en este estudio. Tienen indicación de extracción por el riesgo de perforación de órganos y originar fístulas, abscesos, etc. En los últimos años hemos observado una disminución en la ingestión de alfiler de gancho en lactantes. En una serie previa encontramos que el $14,6 \%$ de los CE eran alfileres de gancho $^{33}$ y en la actual representa el 2,3\%. La frecuencia de ingestión de baterías tipo botón va en aumento, lo que se relaciona con su mayor 
disponibilidad. Al igual que en otras series, estas correspondían a juguetes o comandos de equipos $^{34}$. Cuando las baterías tipo botón están impactadas en esófago hay consenso en la necesidad de extraerlas en forma urgente, idealmente antes de $2 \mathrm{~h}^{34}$; no así cuando estas se encuentran en estómago donde las conductas recomendadas son variables ${ }^{35-39}$. En esta serie hemos observado que las baterías en estómago producen lesiones mucosas en pocas horas, lo que sumado a que no es predecible si se movilizará espontáneamente, nos hace plantear al igual que otros autores la necesidad de su extracción precoz.

Hemos tenido pocos casos de ingestión de imanes, los que generalmente son parte de juegos. Pueden producir daño aún siendo únicos ${ }^{40}$ ya que al adherirse a objetos metálicos externos como hebilla de cinturón pueden llegar a producir una perforación intestinal.

En esta serie, la extracción precoz de los $\mathrm{CE}$ se relaciona con la consulta precoz, la localización del CE y el tipo de CE. En relación a la técnica de extracción es importante disponer de una variedad de pinzas y en lo posible de un cuerpo extraño similar al que se tragó el niño, para poder simular antes de la endoscopia, maniobras de agarre y extracción del CE.

Encontramos una alta frecuencia de lesiones mucosales consideradas complicaciones menores. Estas son por decúbito o por el paso de objetos punzantes, generalmente superficiales, se localizan en esófago en el 76,7\% de los casos y se relacionan con el tipo de CE más que con el tiempo de permanencia del CE. En otras series se reporta un $15 \%$ de complicaciones menores ${ }^{36}$ lo que según sus autores, el bajo porcentaje se debería a una extracción precoz del CE. Pensamos que el alto porcentaje de lesiones mucosales en nuestra serie se debe a una búsqueda dirigida de lesiones ya que el tiempo de extracción también fue precoz. Sólo 9 pacientes requirieron hospitalización para observación, por la severidad del daño de la mucosa, evolucionando en buena forma con tratamiento médico.

Todos los casos se resolvieron por vía endoscópica y no observamos complicaciones derivadas del procedimiento ni de la sedación.

En conclusión, la ingestión de CE es un ac- cidente que ocurre en el hogar, y se presenta con mayor frecuencia en el menor de 6 años. El CE más frecuente es la moneda seguido por las baterías tipo botón. El método diagnóstico más usado es la Rx Simple. La extracción endoscópica del CE, con el paciente en sedación profunda, fue exitosa y sin complicaciones. El dar a conocer los objetos y las circunstancias en que los niños se tragan los $\mathrm{CE}$, nos permitirá alertar a los pediatras para que puedan educar a los padres y cuidadores y disminuir la incidencia de este accidente.

\section{Referencias}

1.- Stevenson RD, Allaire JH: The development of normal feeding and swallowing. Pediatr Clin North Am 1991; 38: 1439-53.

2.- Conners $G$, Chamberlain J, Weiner P: Pediatric coin ingestion: A home-based survey. The American Journal of Emergency Medicine 1995; 13: 638-40.

3.- Bronstein AC, Spyker DA, Cantinela LR: 2009 Annual report of the American Association of Poison Control Centers' National Poison Data System (NPDS) 27 $7^{\text {th }}$ Annual Report. Clin Toxicol 2010; 48: 979-1178.

4.- Wyllie R: Foreign bodies in the gastrointestinal tract. Curr Opin Ped 2006; 18: 563-4.

5.- Conners GP: Pediatrics Foreign body ingestion. Emedicine Jul 12, 2010.

6.- Aktay AN, Werlin SL: Penetration of the stomach by an accidentally ingested straigh pin. J Pediatr Gastroenterol Nutr 2002; 34: 81-2.

7.- Pickard LR, Tepas JJ, Agarwal BL, Haller JA: Duodeno-renal fistula: An uncommon complication of an ingested foreign body. J of Pediatr Surg 1980; 15: 337 8.

8.- Beer S, Avidan G, Viure E, Starinsky R: A foreign body in the oesophagus as a cause of respiratory distress. Pediatr Radiol 1982; 12: 41-2.

9.- Bocquet N, Guillot L, Mougenot JF, Ruemmele FM, Cheron $G$ : Hematemesis in an 11 month child: an unusual symptom revealing a foreign body in the stomach. Arch Pediatr 2005; 12: 424-6.

10.- Chen $M K$, Bejerle EA: Gastrointestinal foreign bodies. Pediatr Ann 2001; 30: 736-42.

11.- Nandi P, Ong GB: Foreign body in the oesophagus: review of 2394 cases. Br J Surg 1978; 65: 5-9.

12.- Pak MW, Lee WC, Fung HK, van Hasselt A: A prospective study of foreign-body ingestion in 311 children. 
International J Paediatrics Otorhinolarimgology 2001; 58: $37-45$.

13.- Lakdhar-Idrissi M, Hida M: Foreign body ingestion in children: 105 case reports. Arch Pediatr 2011; 18: 85662.

14.- Aydogdu S, Arikan C, Çakir M, et al: Foreign body ingestion in Turkish children. The Turkish J Pediatr 2009; 51: 127-32.

15.- Ikenberry $S$, Jue T, Anderson $M$, et al: Standards of Practice Committee. American Society for Gastrointestinal Endoscopy Guidelines for the management of ingested foreign bodies. Gastrointest Endosc 2011; 73: 1085-91.

16.- Uyemura $M C$ : Foreign body ingestion in children. Am Fam Physician 2005; 72: 287-91.

17.- Arana A, Hauser B, Hachimi-Idrissi S, Vandenplas Y: Management of ingested foreign bodies in childhood and review of the literature. Eur J Pediatr 2001; 160 : 468-72.

18.- Cheng W, Tam PKH: Foreign Body ingestion in children: Experience with 1265 cases. J Pediatr Surgery 1999; 34: 1472-6.

19.- Panieri E, Bass DH: The management of ingested foreign bodies in children- a review of 663 cases. Eur J Emerg Med 1995; 2: 83-7.

20.- Louie JP, Alpern ER, Windreich RM: Witnessed and unwitnessed esophageal foreign bodies in children. Pediatr Emerg Care 2005; 21: 582-5.

21.- Paul R, Christoffel K, Binns H, Jaffe D: Foreign body ingestions in children: Risk of complication varies with site of initial health care contact. Pediatrics 1993; 91: 121-7.

22.- Gregori D, Scarinzi C, Morra B, et al: Ingested foreign bodies causing complications and requiring hospitalization in European children: Result from ESFBI study. Pediatrics International 2010; 52: 26-32.

23.- Gómez de Terreros I, Gómez de Terreros M, Serrano Santamaría $M$, et al: Ingestión reiterada de cuerpos extraños. Forma inusual de presentación del síndrome de munchausen por poderes. Child Abuse \& Neglet 1996; 20: 613-20.

24.- Nolte KB: Esophageal Foreign Bodies as Child Abuse: Potential Fatal Mechanisms. Am J Forensic Med Pathol 1993; 14: 323-6.

25.- Tokar B, Cevik AA, Ilhan EH: Ingested gastrointestinal foreign bodies: predisposing factors for complications in children having surgical or endoscopic removal. Pediatr Surg Int 2007; 23: 135-9.

26.- Hurtado CW, Furuta GT, Kramer RE: Etiology of esophageal food impactions in children. J Pediatr Gastroenterol Nutr 2011; 52: 43-6.

27.- Kerlin P, Jones D, Remedios M, Campbell C: Prevalence de eosinophilic esophagitis in adult with food bolus obstruction of the esophagus. J Clin Gastroenterol 2007; 41: 356-61.

28.- Macphemsoni R, Hill J, Othersen HB, Tagge E, Smith $C$ : Esophageal Foreign Bodies in Children: Diagnosis, Treatment, and Complications. Am J Roentgenol 1996; 166: 919-24.

29.- Bloom R, Nakano P, Gray S, Skandalakis J: Foreign bodies of the gastrointestinal tract. Am Surg 1986; 52: 618-21.

30.- Little DC, Shah SR, St Peter SD, et al: Esophageal foreign bodies in the pediatric population: our first 500 cases. J Pediatr Surg 2006; 41: 914-8.

31.- Waltzman M, Baskin M, Wypij D, Mooney D, Jones D, Fleisher GA: Randomized clinical trial of the management of esophageal coins in children. Pediatrics 2005; 116: 614-9.

32.- Soprano JV, Mandl KD: Four strategies for the management of esophageal coins in children. Pediatrics 2000; 105: 1-5.

33.- Ríos G, Miquel I, Saelzer E: Endoscopic removal of foreign bodies from upper gastrointestinal tract of children. J Pediatr Gastroenterol Nutr 2000; 31(Suppl 2) S 189.

34.- Litovitz T, Whitaker N, Clark L: Preventing battery ingestions: An analysis of 8648 cases. Pediatrics 2010; 125: 1178-83.

35.- Litovitz T, Whitaker N, Clark L, White NC, Marsolek $M$ : Emerging battery-ingestion hazard: clinical implications. Pediatrics 2010; 125: 1168-77.

36.- Lin CH, Chen AC, Tsai J-D, Wei SH, Hsueh KC, Lin $W$-C: Endoscopic removal of foreign bodies in children. Kaohsiung J Med Sci 2007; 23: 447-52.

37.- Takagaki K, Rothbaum E, Folashade AJ, Heyman M: Gastric Mucosal Damage From Ingestion of 3 Button Cell Batteries. J Pediatr Gastroenterol Nutr 2011; 53: 222-3.

38.- Aydogdu S, Arikan Ç, Çakir M, et al: Foreign body ingestión in Turkish children. Turk J Pediatr 2009; 51: 127-32.

39.- Maluenda $C$, Varea $V$, Bodas A: Ingesta de cuerpos extraños. Protocolos diagnóstico-terapéuticos de Gastroenterología, Hepatología y Nutrición Pediátrica. SEGHNP-AEP 2011 Cap 15: 131-4.

40.- Butterworth J, Feltis B: Toy magnet ingestion in children: revising the algorithm. J Pediatr Surg 2007; 42: e3-5. 